\title{
Random connections in memory
}

Neuron https://doi.org/10.1016/j.neuron.2019.04.020

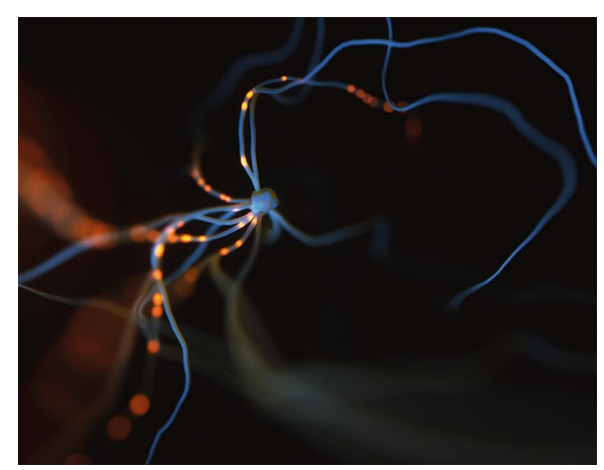

Credit: Panther Media GmbH / Alamy Stock Photo

Working memory is the type of memory that represents information that we currently use to select actions, for example when we enter a phone number that we've just looked up. A long-standing conundrum in working memory research is that working memory has two seemingly contradictory features it is flexible enough to store anything (any phone number) but also has a strict limit on the number of things you can remember at a time (only one phone number at a time).

Flora Bouchacourt and Timothy Buschman at Princeton now present a neural model of working memory that explains this dualism. Past models have for example assumed that the network supporting working memory has recurrent connections that lead to persistent neural activity, or conversely that memory representations are stored in short-term changes to synaptic connections. While the former models are too stable to explain updating, the latter are too flexible to account for limitations in representing many different items.

The new model has two types of network arranged in separate layers, a sensory network that stores representation and a random network that reciprocally connects to it. This elegant variation creates a model that in many respect behaves like humans, including its ability to represent anything, but a limit to the number of different representations it can faithfully store.

Anne-Marike Schiffer

Published online: 28 June 2019

https://doi.org/10.1038/s41562-019-0661-z 